\title{
An audit on surgical management of Tubal ectopic pregnancies and patients' preferences survey.
}

\author{
Pathiraja. RP ${ }^{1}$, Jayawardane MAMM ${ }^{1}$, Silva $\mathrm{KCDP}^{1}$, Srisanjeevan $\mathrm{S}^{2}$
}

\section{INTRODUCTION}

Ectopic pregnancy is a common early pregnancy emergency encountered every day. The prevalence of ectopic pregnancy is around $1 \%-2 \% .{ }^{1}$ The commonest site being the fallopian tubes which account for $95 \%$ of all ectopics., ${ }^{2,3}$ It still contributes to maternal mortality rates in early pregnancy worldwide., ${ }^{4,5}$ Sri Lanka, being a middle income developing country, has been able to maintain good maternal care with a maternal mortality ratio of $31 / 100,000$ live births. Ectopic pregnancy contributed for $2 \%$ of maternal deaths in 2010 .

Early diagnosis of ectopic pregnancies has led to more conservative management options with preservation of the tubes, instead of Salpingectomy. ${ }^{6,7}$ The Laparoscopic approach is preferred over open surgery due to its benefits. ${ }^{8}$ At Present, Patients are not given proper information on their condition and the available management options, their opinions and preferences and their satisfaction following treatment are not routinely assessed.

There is still a debate on whether salpingectomy or salpingotomy be performed even in the presence of an apparently normal contra lateral tube. According to an Epidemiologic Register for ectopic pregnancy in

\footnotetext{
1-Department of Obstetrics and Gynaecology, Faculty of

Medical Sciences, University of Sri Jayewardenepura

2- Colombo South Teaching Hospital, Kalubowila
}

\section{Correspondence:}

Dr Ramya Pathiraja, Senior Lecturer, Department of Obstetrics and Gynaecology, University of Sri Jayawardenapura, email: ramya_pathiraja@yahoo. com

E-mail: ramya_pathiraja@yahoo.com

Competing interests: None
Auvergne in France, conservative surgery is considered even when the contra lateral tube is apparently normal as it may improve the chance of fertility. ${ }^{9}$

In two multicenter randomized control studies, there is no significant difference between Salpingectomy or Salpingotomy in terms of increased risk of recurrent ectopic pregnancy and subsequent fertility in medical, conservative surgical and radical surgical groups. ${ }^{10,11}$ Therefore, in the absence of clear advantage or disadvantage in conservative Vs radical surgical management of ectopic pregnancy, the patients preference should play a major role in determining the treatment modality even with a macroscopically normal contra lateral tube. The aim of this audit is to ascertain whether the management of tubal ectopic pregnancy conforms to the guidelines set forth by the Royal College of Obstetricians and Gynaecologists of the United Kingdom and also to assess patient satisfaction and their preferences and whether such preferences were accommodated in decision making and the management of ectopic pregnancy, if they were educated and well-informed on current available data.

\section{METHODS}

This is a retrospective audit on outcomes of the management of tubal ectopic pregnancy. Patients' Bed Head Ticket (BHT) records were reviewed. Patient's information on age, parity and the number of children, period of gestation at presentation was collected. The mode of entry in to peritoneal cavity (Laparotomy or Laparoscopy), type of surgery (conservative salpingostomy or salpingectomy); amount of haemoperitoneum, if present; the state of the ipsi-lateral tube at the time of surgery were acquired from operation notes. A telephone survey was performed by a one trained research assistant using a validated questionnaire which include, her educational status, the amount of information she received regarding her condition, whether she was offered the option of preserving the tube, if possible and her satisfaction regarding the care provided at the hospital and her return to normalcy. All patients who were surgically treated for ectopic pregnancies, with an apparently normal contra lateral tube over a period of 4 months, between $1^{\text {st }}$ November 2013 and $1^{\text {st }}$ March 2014 were included in this audit. Those who had an apparently abnormal contra lateral tube or those who had surgery following failed medical management were excluded.

\section{RESULTS}

A total of 24 patients were surgically treated during the period of 6 months. Two patients were not contactable. One patient had refused to take part in the audit whilst another patient had an abnormal contra lateral tube. Therefore, a total of 20 patients who underwent surgical treatment for ectopic pregnancies, were recruited.

$55 \%$ of patients were between 20 and 30 years of age. There were $3(15 \%)$ women over 36 years of age. $90 \%$ of women were educated up to or beyond the GCE Ordinary Level, they had received secondary education. Only 3 women (15\%), have received undergraduate education.

$40 \%$ of women $(n=8)$ were in their first pregnancy when diagnosed with an ectopic. $30 \%$ of women had a parity of 3 or more. 9 women $(45 \%)$ did not have any children upon diagnosis, 8 being primiparous whilst one mother had a miscarriage in her $1^{\text {st }}$ pregnancy. 5 patients who presented to the 
Table 1. Mode of access to peritoneal cavity in relation to amount of haemoperitoneum

\begin{tabular}{|l|l|l|}
\hline Mode of access & Number $(\mathbf{n})$ & Mean Haemoperitoneum \\
\hline Laparotomy & 7 & $842.86 \mathrm{ml}$ \\
\hline Laparoscopy & 13 & $161.54 \mathrm{ml}$ \\
\hline Total & 20 & \\
\hline
\end{tabular}

gynaecology ward were uncertain of the last regular menstrual period. The average period of amenorrhoea of the patients with a known last menstrual period was 5.8 weeks. Only one patient presented in her $10^{\text {th }}$ week of period of amenorrhoea.

At the time of surgery, 6 patients $(30 \%)$ had very minimal haemoperitoneum. $45 \%$ of patients were found to have a haemoperitoneum of $100 \mathrm{ml}$ lorless. $35 \%$ of patients had a haemoperitoneum of $500 \mathrm{ml}$ or more and only 3 patients had more than $1000 \mathrm{ml}$ of blood in the peritoneal cavity. The mean volume of haemoperitoneum at the time of surgery was $400 \mathrm{ml}$. All were tubal ectopic with right tube involvement in $65 \%$ of patients. Patients in which a ruptured tubal ectopic was diagnosed, laparotomy was the preferred mode of access to the peritoneal cavity and laparoscopic approach was used only in $35 \%$ of patients. When considering the relationship between the amount of haemoperitoneum and the mode of access to the peritoneal cavity, in patients who underwent a laparotomy, a mean haemoperitoneum of 842.86 $\mathrm{ml}$ was observed and in those who underwent a laparoscopy, a mean haemoperitoneum of $161.54 \mathrm{ml}$ was observed. A mean difference of 681.32 $\mathrm{ml}$ was noted and this was found to be statistically significant $(\mathrm{p}=0.001)$. Fallopian tube is already ruptured in $50 \%$ of patients at the time of surgery and the majority $(85 \%)$ has undergone salpingectomy and only $15 \%$ has undergone conservative surgery. 7 patients with un-ruptured tube has also undergone salpingectomy.

Following a telephone survey after discharge, all patients reported that they had returned to normal day to day activities without any hindrance by 8 weeks after surgery, irrespective of the type of surgery. $85 \%$ of patients rated the care provided by the hospital as "very good" or "excellent". All patients were satisfied with the care provided and that the information received regarding her condition was adequate. $95 \%$ of patients perceived that the information provided regarding the ectopic pregnancy was either "good", "very good" or "excellent".

$75 \%$ of patients claimed that they were not given the option of preserving the tube, prior to surgery and only $25 \%$ claimed that the option of conservative surgery was offered to them. Though when asked "what would you prefer, if you were informed that according to current available evidence, preservation or removal of the fallopian tube would not alter the chance of becoming pregnant nor the chance of having a repeat ectopic pregnancy significantly", $45 \%$ of patients had stated that they would have considered conservative tube preservation surgery. $10 \%$ of patients $(n=2)$ stated they would opt for a salpingectomy. yet equally, $45 \%$ of patients wanted the doctor to decide on what was best.

\section{DISCUSSION}

The advantages of performing laparoscopic surgery for the treatment of ectopic pregnancy are well documented. The current debate is whether to perform conservative surgery or radical surgery. In the absence of statistically significant improvements in subsequent fertility rates, and the risks of recurrent ectopic pregnancy rates shown by RCTs 10,11, patient's preference and individualized care appears to play an important role, despite popular recommendation of salpingectomy in the presence of macroscopically normal contralateral tube. This was also supported by the outcomes showed by the ectopic population register in France. Though this trend is followed in particular in Europe, in Sri Lanka, being a developing country, the option of laparoscopic surgery could restraints in resources and expertise. not be provided to all women due to
Majority of women were young and educated in this study reflecting the high literacy rate of the country and this would have influenced them to select the option to preserve their organs. In all women diagnosis was made early .The average period of amenorrhea was 5.8 weeks with a mean of 6 weeks.

Themeanvolumeof haemoperitoneum found at the time of surgery was $400 \mathrm{ml}$ with a range between $0 \mathrm{ml}$ to $1500 \mathrm{ml}$ with $50 \%$ of the population had haemoperitoneum of less than $200 \mathrm{ml}$. Early diagnosis, in the absence of dedicated early pregnancy units, is evident, however there is still scope for improvement such as an early pregnancy assessment unit. Though $50 \%$ of patients were found to have an un-ruptured tube at the time of surgery, enabling them to undergo conservative surgery if given the option, a ruptured tubal ectopic does not necessarily rule out the possibility of a conservative surgical approach.

Only $65 \%$ of the patients had a laparoscopic approach which is still below the standard. The mean volume of haemoperitoneum at the time of surgery was statistically significant $(p=0.001)$ when compare to laparotomy over laparoscopy. However other factors such as, the time of presentation to the hospital, the unavailability of laparoscopic facilities at night and the expertise and confidence of the on call team in performing surgery for ectopic pregnancy may have also contributed to the decision of open surgery over Laparoscopy which was not assessed in this audit.

All women who were recruited and who subsequently underwent surgery had an apparently normal contralateral tube and hence, according to the guidelines set forth by the Royal College of Obstetricians and Gynaecologists $^{12}$ salpingectomy was the preferred surgery. In our audit tubal rupture was not the main contributor to a higher percentage of radical surgery and the option of salpingostomy could have been offered if circumstances permitted and patients' preference was considered. $95 \%$ of patients rated the information 
provided about their condition was "good", "very good" or "excellent" but the tubal preservation was offered only to $25 \%$ of the patients.

Retrospectively, when the options of both conservative and radical treatment is offered, in the back ground of no additional benefit in terms of recurrent ectopic pregnancies and subsequent intra uterine pregnancy rates $45 \%$ preferred conservative tube preserving surgery (Salpingotomy) and only $2(10 \%)$ preferred the radical surgical approach (Salpingectomy) and $45 \%$ of patients wanted the doctor to decide the best treatment for them. This highlights the importance of the role of the patient in decision making as well as the preference of the clinician in individualizing the management of ectopic pregnancy especially in selective, high risk patients such as those having a history of sub fertility, pelvic infection and advanced age, despite having a contralateral normal tube.

\section{RECOMMENDATIONS AND LIMITATIONS}

The standard of care can be improved by performing laparoscopic surgery. Though, when dealing with tubal ectopic pregnancies with a normal contra lateral tube, the preferences of the patient should also be taken in to consideration and the management should be individualized. The diagnosis of ectopic pregnancies and their subsequent management took place in the absence of early pregnancy units. Though the RCOG green top guidelines suggest radical surgery, the setting up of such units and the availability of laparoscopic facilities would not only reduce patients' morbidity but also broadens the options available.

This audit also highlights the need to maintain an ectopic register. This would further enhance our understanding of morbidity and fertility following management of ectopic pregnancy in local setting. Patient satisfaction is of utmost importance and it is prudent that their opinions need to be included in facilitating a holistic approach to the management of ectopic pregnancies. The limitation of our study is the retrospective nature of the audit and the small sample size and a prospective study with a good sample size should be carried out to get a clear picture of the situation.

\section{REFERENCES}

1. Barnhart KT. Clinical practice. Ectopic pregnancy. N Engl J Med 2009; 361: 379-87.

2. Brenner PF, Raj S, Michell DR Sr. Ectopic pregnancy: A Study of 300 consecutive surgically treated cases. JAMA 1980;243:673

3. Boronow RC, McElin TW, West RH, et al. Ovarian pregnancy: a report of 4 cases and a 13 year survey of English literature. Am J Obstet Gynecol 1965; 91:1095

4. Maymon R, Shulman A, Halperin R, Michell A, Bukovsky I. Review of 197 patients treated by salpingectomy of salpingotomy. Eur J Obstet Gynecol Reprod Bilo 1995;62:61-67
5. Lewis G (ed) Saving mothers lives: Reviewing Maternal Deaths to Make Motherhood safer 2003-2005. The Seventh Report on Confidential Enquiries in to Maternal Deaths in the United Kingdom.

6. Kirk E, Papageorghious AT, Condous G, Tan $L$, et al. The diagnostic effectiveness of a initial trans vaginal scan in detecting ectopic pregnancy. Hum Reprod 2007;22:2814-2828

7. Kadae N, De Vore G, Romero R. Discriminatory hCG zone: its use in the sonographic evaluation for ectopic pregnancy. Obstet Gynecol 1981;58:156161

8. Brumsted J, Kessler C, Gibson C, Nakajima D, Riddick DH, Gibson M. A comparison of laparoscopy and laparotomy for the treatment of ectopic pregnancy. Obstet Gynaecol 1988; 71:889-892

9. Bouyer J, Job-Spira N, Pouly JL et al.Fertility after ectopic pregnancy. Results of the first three years of the Auvergne Register. Contraception Fertilite Sexuaualite. 1996; 24:475-481

10. Femke Mol, Norah $M$ van Mello, Annika Strandell, Karin Strandell, et al. Salpingotomy versus salpingectomy in women with tubal pregnancy (ESEP study): an open-label, multicentre, randomised controlled trial: lancet Published Online February 3, 2014 http://dx.doi.org/10.1016/S01406736(14)60123-9

11. Herve' Fernandez, Perrine Capmas, Jean Philippe Lucot, Benoit Resch et al.Fertility after ectopic pregnancy: the DEMETER randomized trial. Human Reproduction, Vol.28, No.5 pp. 1247-1253, 2013

12. Royal College of Obstetricians and Gynaecologists. The management of tubal pregnancy. Guideline No. 21. London: RCOG Press; 2004, reviewed 2010. 